\title{
Strain induced anomalous red shift in mesoscopic iron oxide prepared by a novel technique
}

\author{
T N NARAYANAN, D SAKTHI KUMAR ${ }^{\dagger}$, YASUHIKO YOSHIDA ${ }^{\dagger}$ and \\ M R ANANTHARAMAN* \\ Department of Physics, Cochin University of Science and Technology, Cochin 682 022, India \\ ${ }^{\dagger}$ Bio-Nano Electronics Research Centre, Department of Applied Chemistry, Toyo University, Japan
}

MS received 29 November 2007; revised 30 July 2008

\begin{abstract}
Nano magnetic oxides are promising candidates for high density magnetic storage and other applications. Nonspherical mesoscopic iron oxide particles are also candidate materials for studying the shape, size and strain induced modifications of various physical properties viz. optical, magnetic and structural. Spherical and nonspherical iron oxides having an aspect ratio, $\sim 2$, are synthesized by employing starch and ethylene glycol and starch and water, respectively by a novel technique. Their optical, structural, thermal and magnetic properties are evaluated. A red shift of $0.24 \mathrm{eV}$ is observed in the case of nonspherical particles when compared to spherical ones. The red shift is attributed to strain induced changes in internal pressure inside the elongated iron oxide particles. Pressure induced effects are due to the increased overlap of wave functions. Magnetic measurements reveal that particles are superparamagnetic. The marked increase in coercivity in the case of elongated particles is a clear evidence for shape induced anisotropy. The decreased specific saturation magnetization of the samples is explained on the basis of weight percentage of starch, a nonmagnetic component and is verified by TGA and FTIR studies. This technique can be modified for tailoring the aspect ratio and these particles are promising candidates for drug delivery and contrast enhancement agents in magnetic resonance imaging.
\end{abstract}

Keywords. Nano magnetic oxides; red shift; magnetic storage.

\section{Introduction}

Mesoscopic magnetic iron oxides have attracted the attention of researchers for a variety of reasons. Magnetic iron oxides are candidate materials for audio, video and compact memories (Hyeon 2003). Recently, efforts are underway to find new applications of these materials - as drug delivery agents, as superparamagnetic iron oxide (SPION) particles for hyperthermia, contrast enhancing agents in magnetic resonance imaging (MRI) (Sabino et al 2004) and also as biosensors (Dallas et al 2006). They are biocompatible and inexpensive. From a fundamental point of view, magnetic iron oxides are ideal templates for studying ferrimagnetism at the nano level since they possess an ideal two sub-lattice with magnetic ions on either sites. Magnetic iron oxides belong to the class of inverse spinels having their cation distributed on the octahedral (B) and tetrahedral (A) sites. Maghemite or gamma ferric oxide also crystallizes in the inverse spinel structure. The structure of maghemite is said to possess a vacancy ordered spinel structure with vacancies situated exclusively on the

*Author for correspondence (mraiyer@gmail.com) octahedral sites (Viswanathan 1990). The preparation of gamma iron oxide from precursors is tricky because the material turns easily into its nonmagnetic phase during synthesis if adequate precautions are not taken.

Nonsphericity is an essential criterion for enhancing signal to noise ratio during recording (Bate 1991; Deng et al 1992). But $\gamma-\mathrm{Fe}_{2} \mathrm{O}_{3}$ is a cubic crystal and does not easily form into elongated ellipsoidal particles. Earlier, researchers attempted to synthesize nonspherical precursors using complexing medium (Anantharaman et al 1997). Nucleation will be greatly influenced by external conditions like $\mathrm{pH}$, temperature and constant stirring. Complexing agents like starch retard the growth of the precursors in the crystallographic $a$-direction, so that growth proceeds in the $c$ direction, leading to needles (Arndt et al 1998). However, with the advent of nanoscience and nanotechnology, shape and size induced modifications of various properties like optical, magnetic, structural are a rich area of physics where quantum mechanical phenomenon like quantum size effects can be investigated on candidate materials like gamma iron oxide (Kachkachi et al 2000). It is also known that size dependent magnetic properties manifest itself into superparamagnetism and single domain characteristics. Reduction of size results in large red shift in $\mathrm{Fe}_{3} \mathrm{O}_{4}$ (Nair et al 2005). 
Most of our earlier studies on nonspherical particles are limited to the synthesis of nonspherical $\gamma-\mathrm{Fe}_{2} \mathrm{O}_{3}$ particles. Most of the particles lie well above the nanometer dimension (Anantharaman et al 1997). However, size, shape and strain dependent optical and magnetic properties are a virgin area where the effect of size and shape can be studied in a single shot if nanosize particles can be synthesized. Such a study assumes importance because of the resulting magneto optical properties of the nanocrystals.

In this work, spherical and elongated mesoscopic gamma iron oxide particles are synthesized employing a novel technique and using starch/water/ethylene glycol as complexing agents. Their structural, magnetic and optical properties are evaluated. Emphasis is laid in studying the shape induced optical properties of gamma iron oxide nanoparticles. Thermogravimetric (TG) and FTIR analysis are carried out to probe the retention of organic residues in the sample. Attempts are made to correlate the optical and magnetic properties with size and shape.

\section{Experimental}

\subsection{Preparation of spherical $\gamma-\mathrm{Fe}_{2} \mathrm{O}_{3}$ nanoparticles}

In order to prepare spherical $\gamma-\mathrm{Fe}_{2} \mathrm{O}_{3}$ particles, $20 \%$ of starch solution was prepared by adding $20 \mathrm{~g}$ of soluble starch into $100 \mathrm{cc}$ of hot water. The resultant solution was heated with constant magnetic stirring to $50^{\circ} \mathrm{C}$ till it became transparent. $25 \mathrm{~g}$ of anhydrous ferrous sulphate was dissolved in $75 \mathrm{cc}$ starch solution. This solution was heated to $70^{\circ} \mathrm{C}$. To this solution 0.2 molar ethylene glycol was added. It was presumed that ethylene glycol inhibits growth of the particles along one particular direction. Addition of ethylene glycol was done drop-wise with constant stirring in order to ensure the formation of spherical particles. These particles were filtered off and dried in a furnace at $370^{\circ} \mathrm{C}$ in ambient atmosphere to ensure the formation of $\gamma-\mathrm{Fe}_{2} \mathrm{O}_{3}$ (Venkatesh Rao et al 1974). This led to a brown magnetic powder confirming the formation of $\gamma-\mathrm{Fe}_{2} \mathrm{O}_{3}$ nanoparticles (Khaleel 2004). These samples are hereafter named $\mathrm{N}-1$.

\subsection{Preparation of ellipsoidal gamma iron oxide}

The above procedure for the synthesis of spherical iron oxide particles was modified to prepare ellipsoidal gamma iron oxide (hereafter named N-2) in the sense, that instead of ethylene glycol and starch, water and starch are employed as the complexing agents for this medium. $25 \mathrm{~g}$ of anhydrous ferrous sulphate was dissolved in $75 \mathrm{cc}$ starch solution. This solution was heated to $70^{\circ} \mathrm{C}$. To this solution 0.2 molar solution of oxalic acid was added to precipitate nonspherical ferrous oxalate precursors from the medium. It was found that as the concentration of starch increases aspect ratio also increases.

\subsection{Characterization}

The structural analysis and the identification of the phase were conducted with an X-ray diffractometer (Rigaku Dmax-C) using $\mathrm{CuK} \alpha$ radiation ( $\lambda=1.5418 \AA$ ). A scanning rate of $5^{\circ} \mathrm{min}^{-1}$ was applied in the $2 \theta$ range of $10^{\circ}-70^{\circ}$. The grain size was estimated by employing DebyeScherrer's formula

$$
D=0.9 \lambda / \beta \cos \theta,
$$

where $\lambda$ is the wavelength of X-ray used, $\beta$ the FWHM of XRD peak with maximum intensity and $\theta$ the angle of diffraction. A JOEL JEM 2200 FS electron microscope using an accelerating voltage of $200 \mathrm{kV}$ was used to deduce the particle size distribution of nanoparticles. The transmission electron microscope (TEM) images, high resolution transmission electron microscope (HRTEM) images and energy dispersed spectra (EDS) were taken for morphological and compositional studies. Planes were identified from HRTEM. Room temperature magnetic properties were obtained from the hysteresis loop recorded in a vibrating sample magnetometer (VSM) model EG\&G Par 4500. A Thermo Nicolet Avatar 370 DTGS model spectrophotometer using $\mathrm{KBr}$ method was employed to record the FTIR spectrum of the samples. A Jasco V 530 UV-visible spectrophotometer was used to determine the diffused reflectance studies. DTG studies were conducted using Perkin Elmer Thermal Analysis in nitrogen atmosphere in the temperature range $30-550^{\circ} \mathrm{C}$.

\section{Results and discussion}

\subsection{Structural and morphological studies using XRD and TEM}

The X-ray powder diffraction pattern for the spherical and nonspherical maghemite particles are depicted in figure 1. They represent a spectrum typical of an inverse spinel.

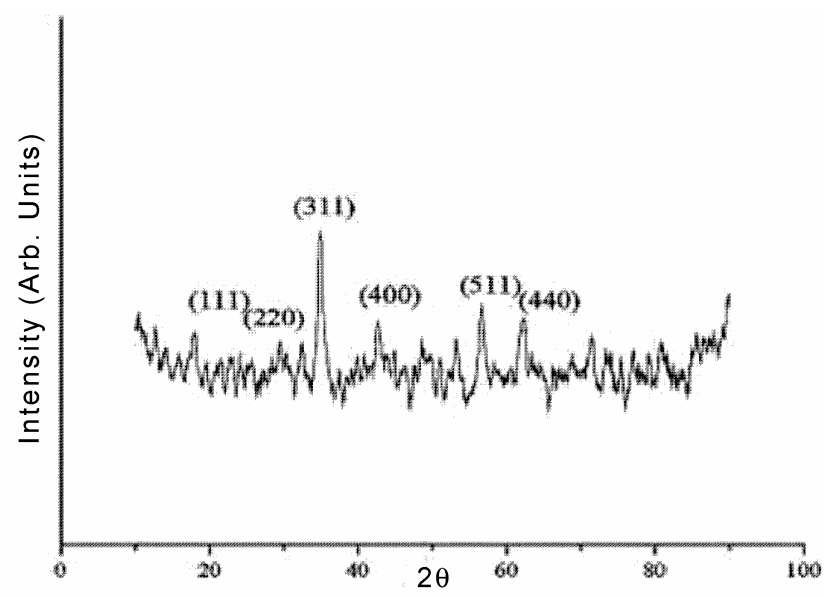

Figure 1. XRD pattern of $\gamma-\mathrm{Fe}_{2} \mathrm{O}_{3}$. 
(a)

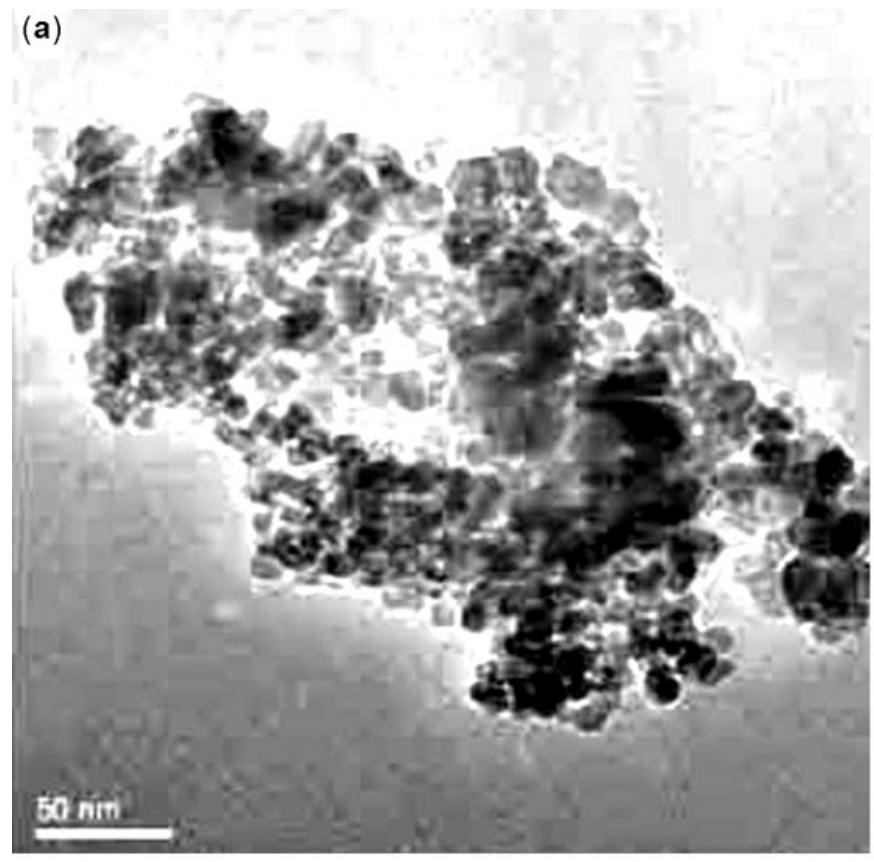

(c)

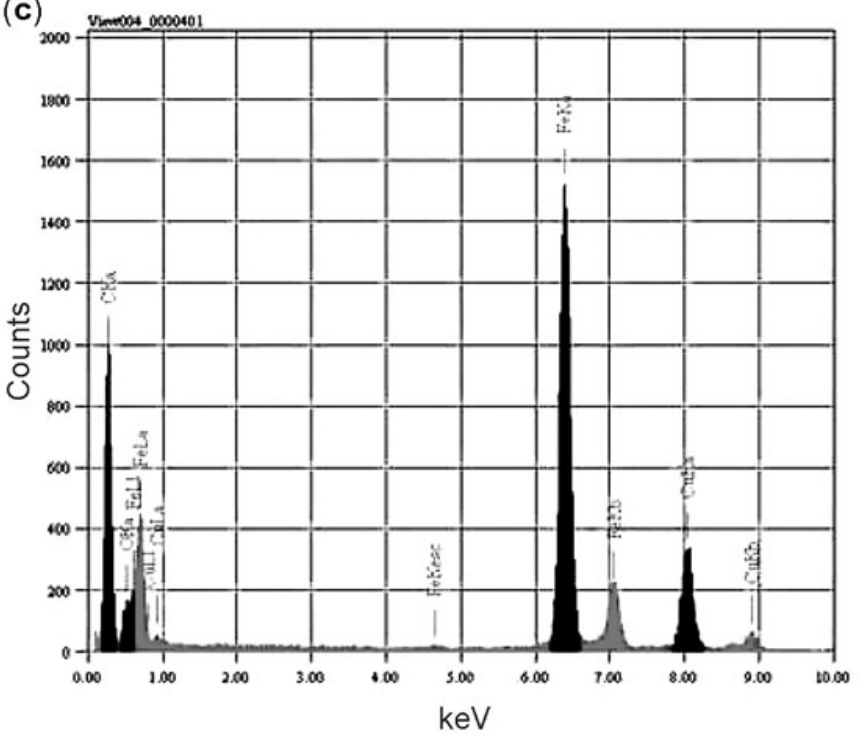

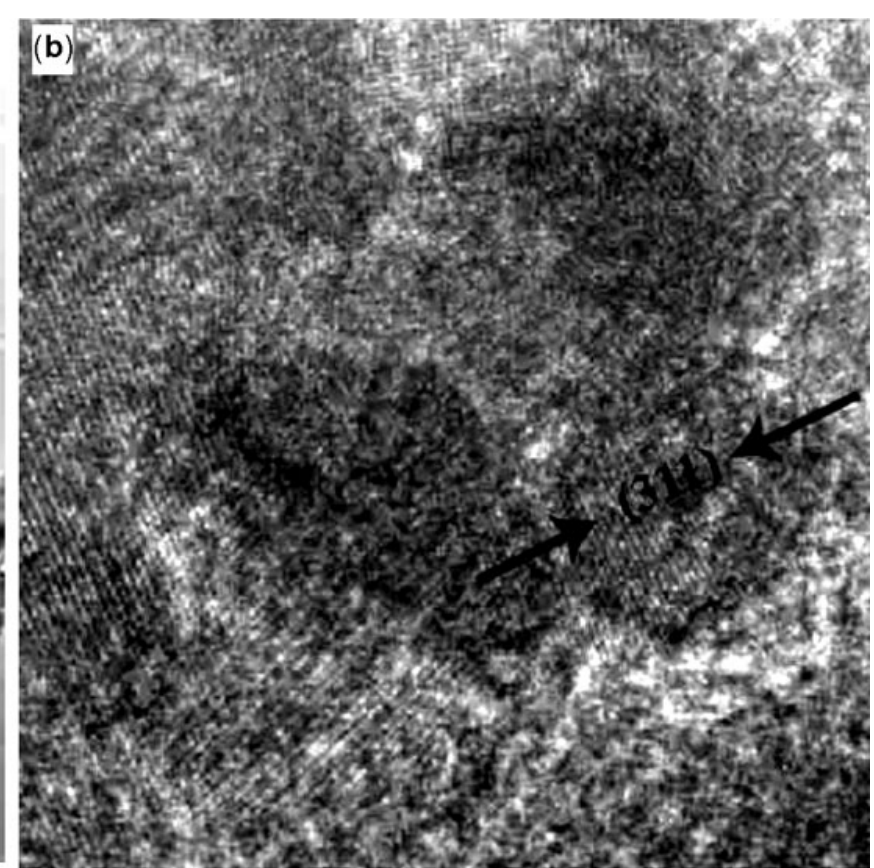

(d)

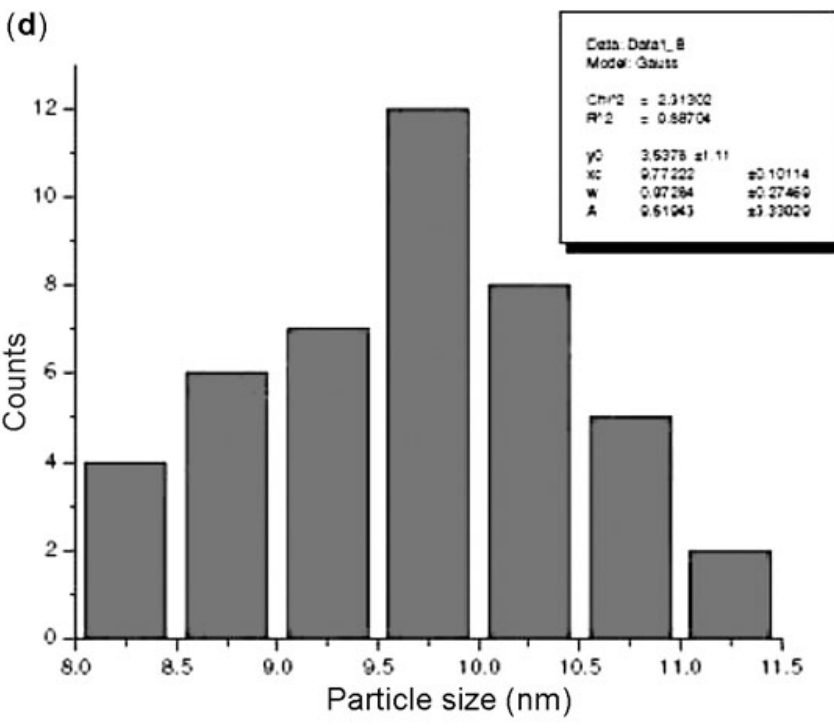

Figure 2. (a) TEM, (b) HRTEM, (c) EDS and (d) size distribution histogram images of N-1.

The planes are identified and listed. The peaks (111), (220), (311), (400), (511) and (440) correspond to that of $\gamma-\mathrm{Fe}_{2} \mathrm{O}_{3}$ (ICDD: 39-1346).

There are no indications of the existence of any impurity phase, corresponding to $\alpha-\mathrm{Fe}_{2} \mathrm{O}_{3}$. However, the base line is found to be shifted due to the presence of amorphous phase probably due to the organic inclusions in the samples. The average particle size was determined from line broadening and the spherical particles have an average size of $10 \mathrm{~nm}$ while the nonspherical particles exhibited an average size of $13.5 \mathrm{~nm}$. TEM images of N-2 indicate the formation of nonspherical particles, while particles coded $\mathrm{N}-1$ are spherical in shape (figure 2). The spherical particles have an average size of $10 \mathrm{~nm}$. Nonspherical parti- cles have an average length of $19 \mathrm{~nm}$ (figure 3a) and breadth $9 \mathrm{~nm}$ with an average aspect ratio of 2 .

It is evident from figure $3 b$ that the particles are crystalline and well separated by grain boundaries. Diffusion of lattice planes into the grain boundary is also noted. The particle size distribution for spherical particles is determined and a histogram depicting the size distribution is shown in figure $2 \mathrm{~d}$. The discrepancy in particle size as determined by XRD and TEM could be because of the fact that only large crystallites contribute to the Braggs peak (Cohen 1990). It can be seen from figure 3b that although the crystal lattice of particles are well defined, the Miller indices are interlocked (buckled) and lattice planes have a small bending in the grain boundary. These 

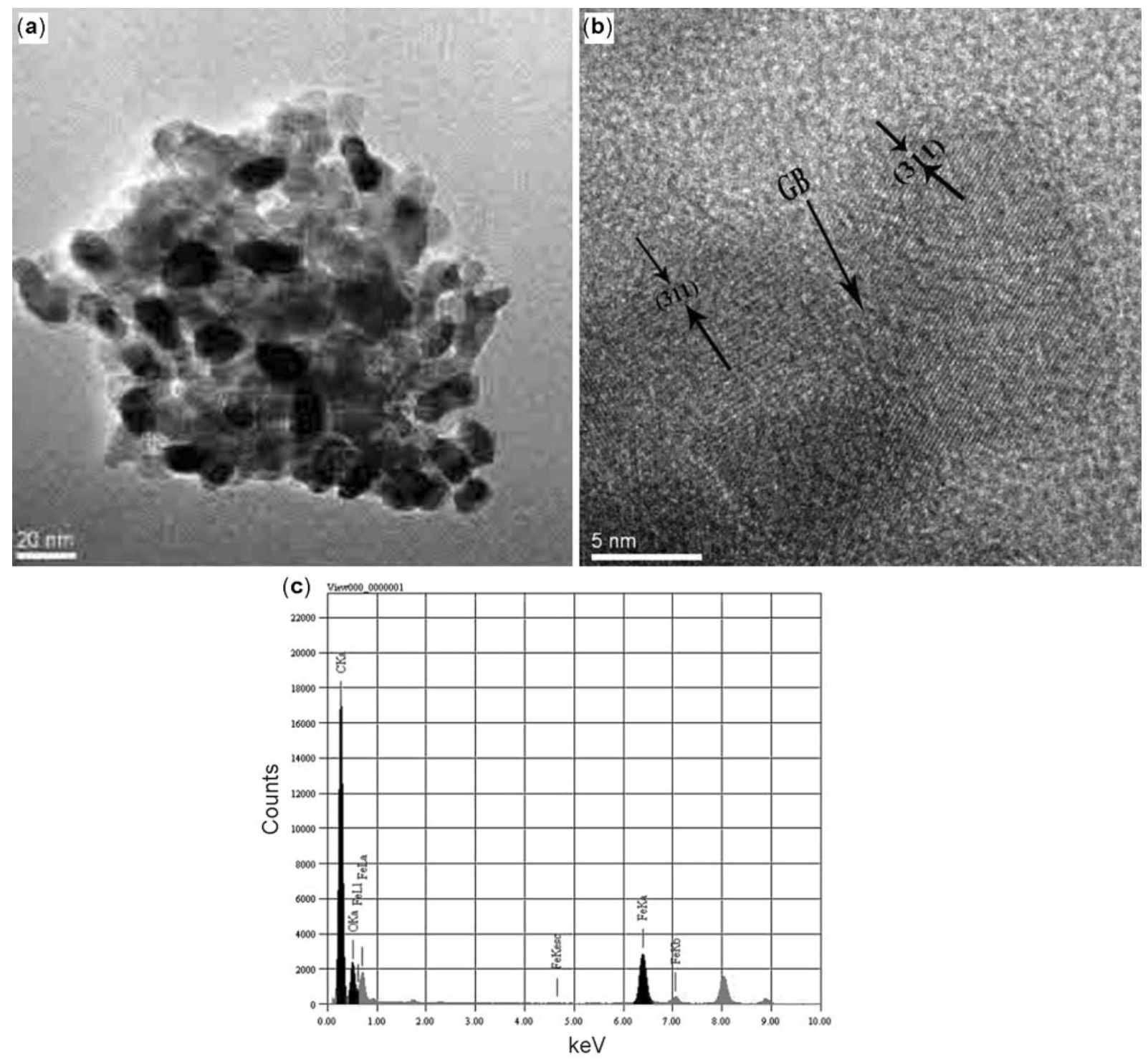

Figure 3. (a) TEM, (b) HRTEM and (c) EDS images of N-2.

are evidence for the occurrence of high internal strain within the lattice (Vassiliou 1994).

\subsection{Magnetization studies using vibrating sample magnetometer (VSM)}

The hysteresis loop for spherical (N-1) and nonspherical iron oxide particles $(\mathrm{N}-2)$ are shown in figures 4 and 5 . Nonspherical particles exhibit a saturation magnetization of $30 \mathrm{emu} / \mathrm{g}$ whereas spherical particles display a saturation magnetization of $37 \mathrm{emu} / \mathrm{g}$ and a coercivity of 105 Oe. Enhancement of coercivity for sample N-2 and decrease in its saturation magnetization with respect to $\mathrm{N}-1$ are other evidences for the deviation from sphericity of sample N-2. If the particles are spherical, the same applied field would have magnetized it to the same ex- tent. If it is not, the same applied field will magnetize it along a long axis. Hence the strength of the applied magnetic field along the short axis is such that it produces the same true field inside the specimen. Thus shape alone is the source of magnetic anisotropy (Cullity 1972). Thus it can be inferred from the magnetization curves of $\mathrm{N}-1$ and $\mathrm{N}-2$ that increase in coercivity in the case of $\mathrm{N}-2$ is mainly due to the elongation of grains. These ellipsoidal particles may cause a small amount of spin freezing in random directions. This is one of the reasons attributable to the decrease in saturation magnetization value for $\mathrm{N}-2$. The magnetization curve of $\mathrm{N}-1$ shows almost negligible remanance and small coercivity showing their superparamagnetic nature.

Additional evidence for superparamagnetism exhibited by N-1 can be obtained by simulating the magnetization 
curve using the Langevin function, $L(x)$. Here it is assumed that the individual grains are single crystals with the number of crystals equal to the number of domains.

We have Langevin function represented by

$$
L(x)=\cot h x-\frac{1}{x}
$$

Size plays a crucial role in deciding the overall magnetization. The variation of particle size is then to be taken into account. The size distribution obtained from TEM is used for simulation. After giving due provision for $b$ (width of the size distribution histogram), the Langevin function can be modified as (Chikazumi 1964)

$$
\begin{aligned}
L(x) & =\frac{1}{2 b x} \int_{x(1-b)}^{x(1+b)} L\left(x^{\prime}\right) \mathrm{d} x^{\prime} \\
& =\frac{1}{2 b x} \ln \left\{\frac{(1-b) \sin h[x(1+b)]}{(1+b) \sin h[x(1-b)]}\right\} .
\end{aligned}
$$

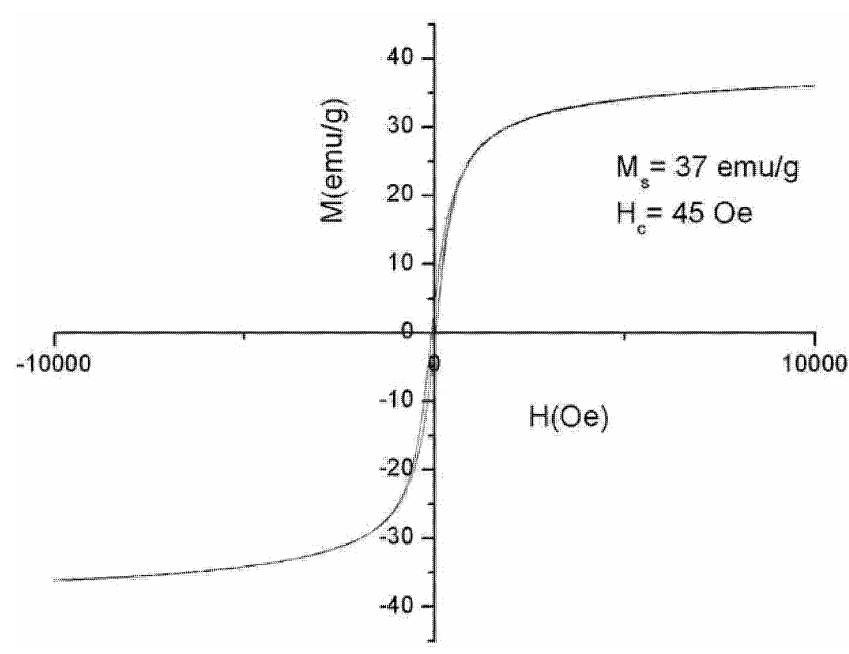

Figure 4. Magnetic hysteresis loop for N-1.

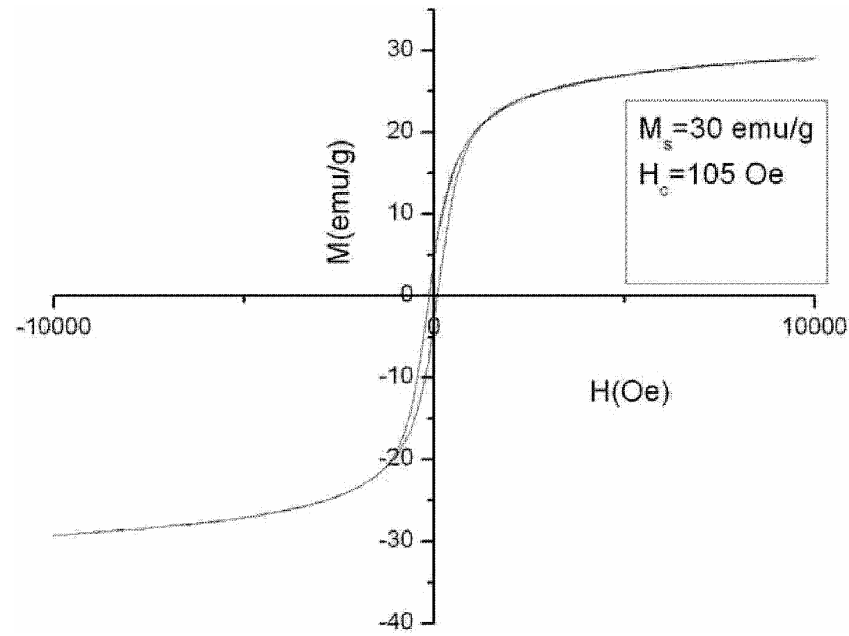

Figure 5. Magnetic hysteresis loop for N-2
The simulated Langevin function using (4) is carried out and is shown in figure 6. The fitting (figure 6) is carried out after giving due weightage for particle size distribution. Thus simulated curve (linear) fits very well with the experimental curve (dotted).

\subsection{DTG-TG studies}

Bulk maghemite in the micron regime possesses a saturation magnetization of $\sim 74 \mathrm{emu} / \mathrm{g}$ (Cullity 1972) and coercivity of 250 Oe (Venkatesh Rao et al 1974), and they are generally multidomain in character. However, as particle size reduced they became single domain and exhibited superparamagnetic characteristics. Although we have procured evidence for superparamagnetism by Langevin fitting, the reduction in magnetization is to be probed by supplementary techniques. For this TG-DTG studies are carried out. The TG-DTG curves are shown in figure 7.

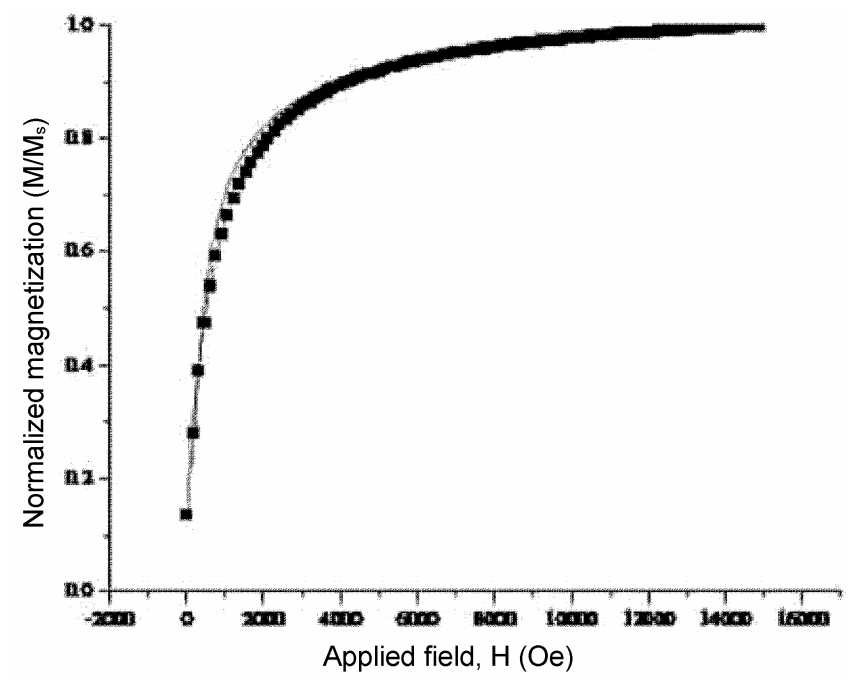

Figure 6. Langevin function fit for N-1

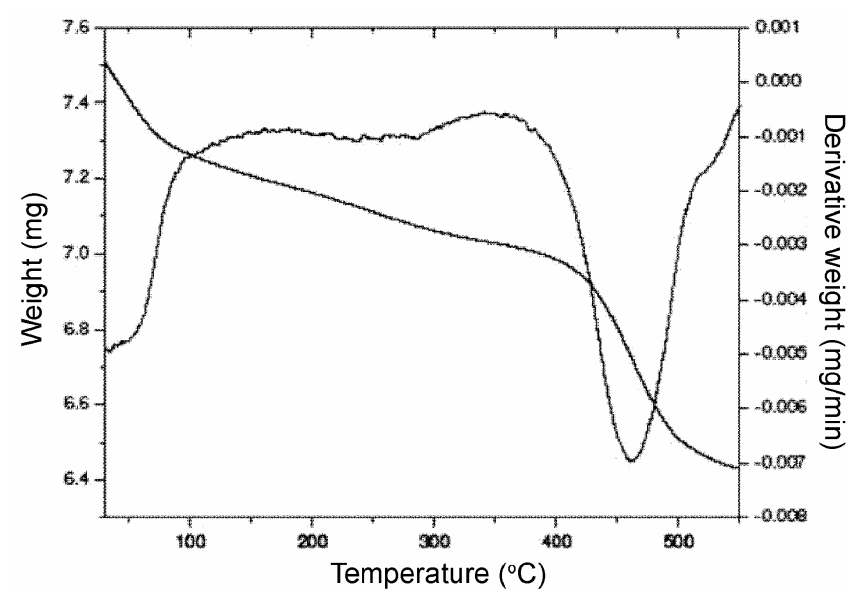

Figure 7. TG-DTG curves for N-1. 
TG curve shows a continuous weight loss on heating. A substantial weight loss corresponding to $8 \%$ at $450^{\circ} \mathrm{K}$ is observed. The net weight loss is around $14 \%$ when the temperature reaches $550^{\circ} \mathrm{K}$. There is a possibility of a small fraction of the polymer (particularly starch which can remain there till $1000^{\circ} \mathrm{C}$ ) being retained in the sample which normally does not decompose at these temperatures. If due weightage is provided to the retention of polymeric residues, in the final gamma ferric oxide the observed saturation magnetization can be accounted for.

\section{$3.4 \quad F T-I R$ studies}

FT-IR studies are carried out on N-1 and N-2 samples in order to ascertain the presence of starch. The spectrum is charted in figure 8 .

The peak at 3384.82 corresponds to - $\mathrm{OH}$ group and the broadening shows the presence of hydrogen bonding. This strengthens the evidence for the presence of starch

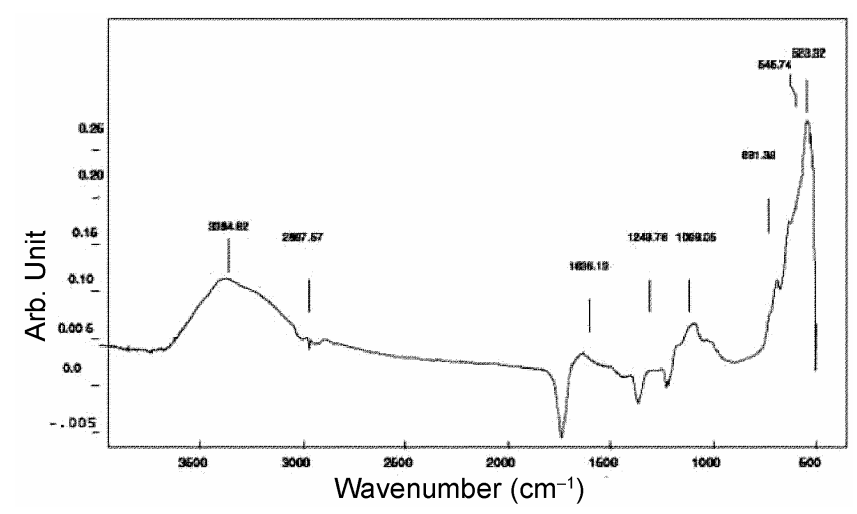

Figure 8. FT-IR spectrum for $\mathrm{N}-1(\mathrm{~N}-2)$.

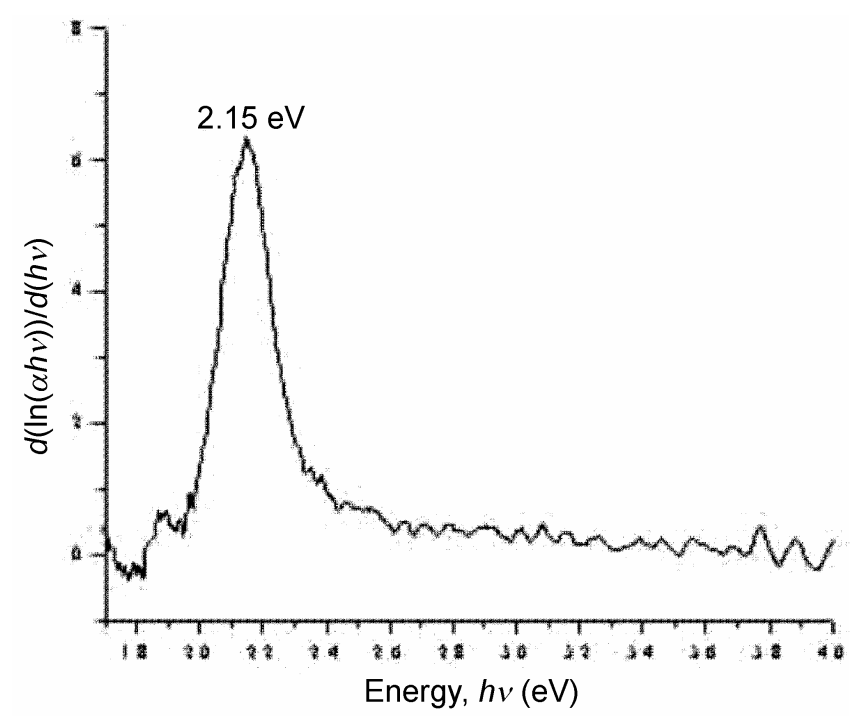

Figure 9. Bandgap calculation from differential plot for N-1. in the samples (Silverstein and Webster 1964). This $-\mathrm{OH}$ group with hydrogen bonding is coming from starch. Peaks between 400 and $700 \mathrm{~cm}^{-1}$ correspond to $\mathrm{Fe}-\mathrm{O}$ bonds (Raksha Sharma et al 2005).

\subsection{Energy bandgap calculation}

Diffused reflectance spectroscopic (DRS) studies of samples $\mathrm{N}-1$ and $\mathrm{N}-2$ are conducted using UV-visible NIR spectrophotometer. Energy band calculations are carried out using the relation (Pankov 1971)

$$
\alpha=\sum \alpha=\sum \frac{A_{i}\left(h v-E_{g i}\right)^{m_{i}}}{h v},
$$

where the value of $E_{g i}$ and $m_{i}$ correspond to the energy and the nature of the particular optical transition with absorption coefficient, $\alpha_{i}$. For allowed direct, allowed indirect, forbidden direct and forbidden indirect transitions, the value of $m_{i}$ corresponds to $1 / 2,2,3 / 2$ and 3 , respectively (Bhattacharyya et al 1992). Since the particles are nanocrystalline, the values of $E_{g i}$ and $m_{i}$ are determined without presuming the nature of electronic transition (Chakrabarti et al 2004). Equation (5) can be rewritten in the following form

$$
\frac{d(\ln (\alpha h v))}{d(h v)}=\frac{m}{(h v-E)} .
$$

The plot of $d(\ln (\alpha h v)) / d(h v)$ vs $h v$ will produce discontinuity at a particular value of $h v=E$, where a possible electronic transition can occur corresponding to the bandgap $E=E_{g i}$. Figures 9 and 10 show the above plots for samples $\mathrm{N}-1$ and $\mathrm{N}-2$, respectively.

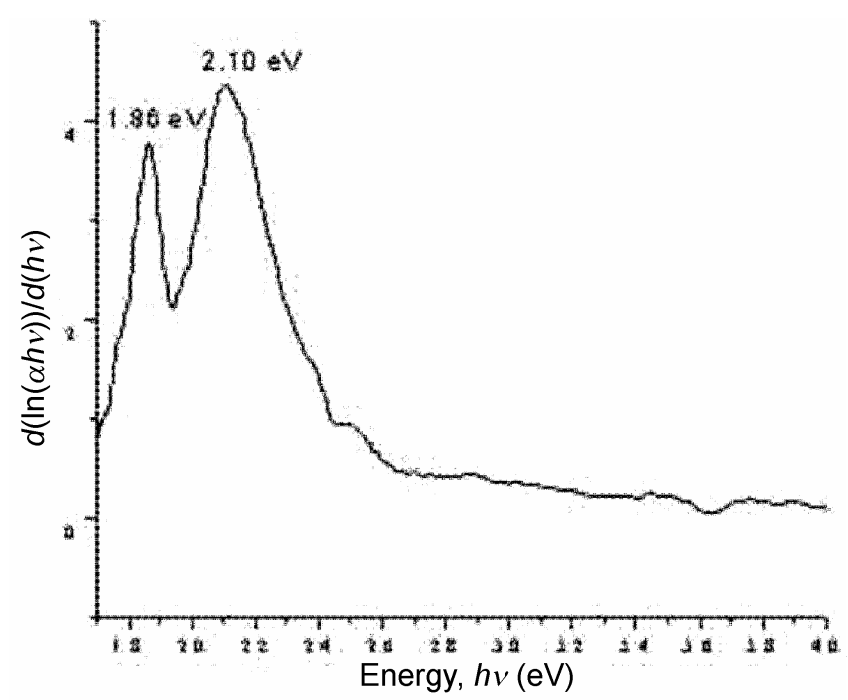

Figure 10. Bandgap calculation from differential plot for N-2. 

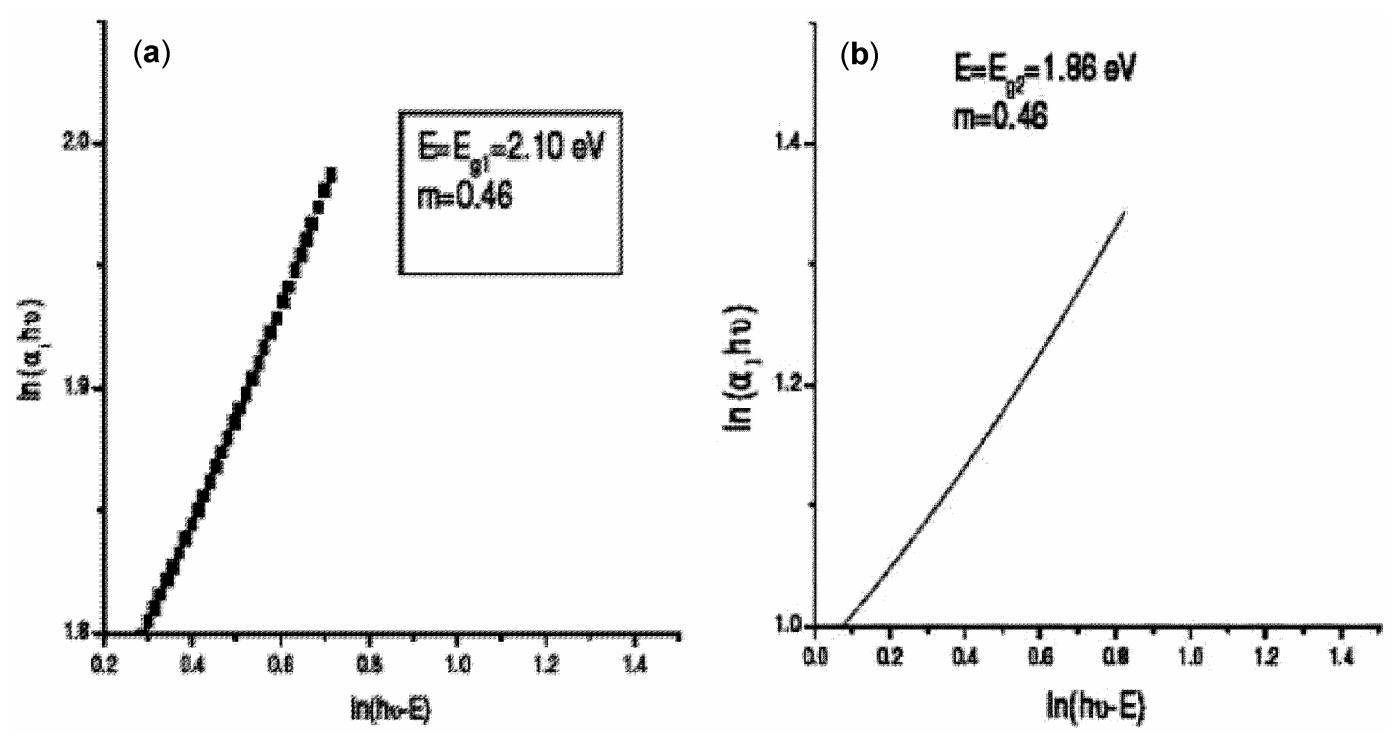

Figure 11. $\ln (h v-E)$ vs $\ln \left(\alpha_{1} h v\right)$ curves for (a) $\mathrm{N}-1$ and (b) N-2.

The nature of electronic transition is allowed direct and it is verified from figures $11 \mathrm{a}$ and $\mathrm{b}(m=0 \cdot 5)$.

The red shifted electronic band $(\sim 0.24 \mathrm{eV})$ is due to the presence of ellipsoidal particles which are under very high stress. This is further supported by HRTEM (figure $3 b)$. This shows that, although the crystal lattice of particles is well defined, the Miller indices are buckled (figure $3 b$ ). From the lattice constants for spherical and nonspherical $\gamma-\mathrm{Fe}_{2} \mathrm{O}_{3}$ particles unit cell compression of $(\Delta V / V)-10 \%$ was observed. Stress increases as the size reduced. The reduction in size is governed by the relation $2 \gamma / r$, where $\gamma$ is pressure inside the grain and $r$ the size of the grain. So strain induced modification of bandgap provides a phenomenological explanation for the anomalous red shift observed in mesoscopic iron oxide particles. The effect of matrix or any other impurities in the red shift has been removed, since the entire measured bandgaps are well above the red shifted value.

\section{Conclusions}

The employment of a complexing medium like starch for the synthesis of nonspherical iron oxide particles can be modified to tailor the aspect ratio of nonspherical nanoparticles of gamma ferric oxide, since shape controlled particles are of interest both from a fundamental and application point of view.

The observed reduction in saturation magnetization with respect to the saturation magnetization of bulk maghemite is due to the presence of superparamagnetism and residual organic impurities in the final product.

Strain induced modification of bandgap is possible by carefully controlling the acicularity of these particles. Shape control can also induce anisotropy and thus modify the coercivity of these particles. A simple phenomeno- logical explanation was provided to account for the red shift found in the optical bandgap of nonspherical maghemite particles.

\section{Acknowledgements}

One of the authors (TNN) thanks the Kerala State Council for Science, Technology and Environment (D.O. No. 004/ FSHIP/05/KSCSTE), Kerala, for financial support in the form of a fellowship and (MRA) thanks the All India Council for Technical Education, Govt. of India (File No. 8023/RID/RPS-73/2004-05).

\section{References}

Anantharaman M R, Joseph K V and Keer H V 1997 Bull. Mater. Sci. 20975

Arndt V, Basf A G and Ludwigshafen 1998 Magn. IEEE Trans. 241796

Bate G 1991 J. Magn. \& Magn. Mater. 100413

Bhattacharyya D, Chaudhuri S and Pal A K 1992 Vacuum 43 313

Chakrabarti S, Ganguli D and Chaudhuri S 2004 Physica E24 333

Chikazumi S 1964 Physics of magnetism (New York: John Wiley \& Sons)

Cohen J B 1990 Ultramicroscopy 3441

Cullity B D 1972 Introduction to magnetic materials (Philippines: Addison-Wesley Publishing Company)

Dallas Panagiotis, Moutis Nicolas, Devlin Eamonn, Niarchos Dimitrios and Pentridis Dimitros 2006 Nanotechnology 17 5019

Deng M C, Hsu S L and Chin T S 1992 IEEE Trans. Magn. 28 2385

Hyeon Taeghwan 2003 Chem. Commun. 927

Kachkachi H, Ezzir A, Nogues M and Tronc E 2000 Eur. Phys. J. B14 681 
Khaleel Abbas A 2004 Chem. Eur. J. 10925

Nair Swapna S, Mathews Mercy and Anantharaman M R 2005 Chem. Phys. Letts 406398

Pankov J J 1971 Optical processes in semiconductors (New Jersey: Prentice-Hall)

Raksha Sharma, Subhalakshmi Lamba and Annapoorni S 2005 J. Phys. D: Appl. Phys. 383354

Sabino Veintemillas-Verdaguer et al 2004 J. Phys. D: Appl. Phys. 372054
Silverstein Robert M and Webster Francis X 1964 Spectroscopic identification of organic compounds (New York: John Wiley \& Sons)

Vassiliou John K, Mehrotra Vivek, Russell Michal W, McMichael R D, Shull R D and Ronald F Ziolo 1993 J. Appl. Phys. 7310 Venkatesh Rao, Shashimohan A L and Biswas A B $1974 \mathrm{~J}$. Mater. Sci. 9430

Viswanathan B 1990 Ferrite materials: Science and Technology (New Delhi: Narosa Publishing House) 